\title{
Análisis de Excelencia educativa en el trienio 2016-2018 para básica secundaria en la Institución Educativa General Santander
}

\section{Analysis of Educational Excellence in the 2016-2018 triennium for basic education at the General Santander Educational Institution}

Jorge Enrique Díaz-Pinzón.

Universidad Nacional de Colombia. iediazp@unal.edu.co

\section{RESUMEN.}

Este trabajo de investigación tuvo por objetivo realizar un análisis de excelencia educativa, Índice Sintético de Calidad Educativa (ISCE), en el trienio 2016 a 2018, en la Institución Educativa General Santander del municipio de Soacha-Cundinamarca, Colombia. Se realizó el cálculo para cada uno de los componentes de excelencia; Progreso, Desempeño, Eficiencia y Ambiente Escolar. Se pudo evidenciar un incremento en su ISCE a partir del año 2016, de 4, 20 hasta llegar en el 2018 a 4, 89. Esto debido a los planes de mejoramiento que la institución ha implementado año tras año para mejorar su ISCE.

\section{PALABRAS CLAVE.}

Educación, Calidad de la Educación, Evaluación comparativa, Excelencia educativa.

\section{ABSTRACT.}

This research work aimed to perform an analysis of educational excellence, Synthetic Index of Educational Quality (ISCE), in the triennium 2016 to 2018, at the General Santander Educational Institution of the municipality of Soacha-Cundinamarca, Colombia. The calculation was made for each of the components of excellence; Progress, Performance, Efficiency and School Environment. It was possible to see an increase in their ISCE from 2016, from 4.20 to 2018 to 4.89 . This is due to the improvement plans that the institution has implemented year after year to improve its ISCE.

\section{KEY WORDS.}

Education, Quality of Education, Comparative Evaluation, Educational Excellence. 


\section{Introducción.}

En Marzo de 2015, el Ministerio de Educación Nacional presentó el Índice Sintético de Calidad Educativa (ISCE) como una herramienta diferente de medición de la calidad educativa en el país.(Zambrano, 2015). El Índice Sintético de Calidad Educativa-ISCE, surgió como una medida sencilla de la calidad de la educación, con el propósito que los niños vayan a la escuela y que aprendan lo que tienen que aprender, esto con el fin de que Colombia sea el país más educado de América Latina en el 2025. (Godoy, 2015). Con ella, sabremos con confianza cómo somos en cada uno de nuestros ciclos educativos -Básica Primaria, Básica Secundaria y Media. (Ministerio de Educación Nacional, 2017)

A partir de los resultados del Índice Sintético de la Calidad Educativa (ISCE), que son una herramienta fundamental para el establecimiento de metas y acciones de progreso, la intrepidez del impacto de las mismas y el involucramiento de la comunidad educativa en esta vía hacia la excelencia. (Jaramillo, 2017). Antes que nada, es importante entender qué es el ISCE. Según el Ministerio de Educación es una herramienta que apoya en la búsqueda de la mejora de todos los colegios. (El Tiempo, 2017).

El Índice está fundamentado por una escala del 1 al 10, siendo 10 el valor más alto que se puede obtener. El puntaje tiene en cuenta cuatro componentes, y, al sumarlos, se obtiene el puntaje total del Índice Sintético de Calidad Educativa (ISCE). (Godoy, 2015)

Según el (Ministerio de Educación Nacional, 2017), el nuevo Índice Sintético de Calidad Educativa se llama "sintético" porque tiene cuatro componentes claves para optimizar son compuestos en uno solo. Estos son:

Progreso: Refleja qué tanto ha mejorado nuestra institución educativa en relación con el año anterior. Este componente da un máximo de 4 puntos de los 10 posibles que un establecimiento educativo puede obtener en el ISCE.

Desempeño: Refleja el puntaje promedio que los estudiantes obtuvieron en las Pruebas Saber 2016 para Matemáticas y Lenguaje, dando un máximo de 4 puntos de los 10 posibles que un establecimiento educativo puede obtener en el ISCE.

Eficiencia: Concierne a la proporción de estudiantes que aprueban el año escolar y pasan al año siguiente. Para primaria y secundaria el valor de eficiencia corresponde al valor reportado por cada colegio en SIMAT sobre su tasa de aprobación (es decir dará máximo 1 punto en el ISCE).

Ambiente escolar: Concerniente a la evaluación de las condiciones propicias para el aprendizaje en el aula de clase. Este componente que máximo dará 1 punto de los 10 posibles que un establecimiento educativo puede obtener en el ISCE.

Según (Bayona, 2016) citado por (Díaz, 2018), el ISCE, extracta en una sola medida cuatro aspectos de la calidad educativa: Progreso, Desempeño, Eficiencia y Ambiente Escolar. La escala del ISCE es de 1 a 10 donde Progreso, centrado como las variaciones (mejoramiento) en el porcentaje de estudiantes en el nivel insuficiente de la prueba, pesa 40\%; Desempeño también pesa $40 \%$ y se calcula dividiendo las pruebas de matemáticas y lenguaje el cual se convierte a un puntaje de 1 a 4; eficiencia pesa el $10 \%$ en primaria y en media el $20 \%$, esta se mide como la tasa de aprobación. En conclusión, ambiente escolar pesa 10\% y solo se mide en primaría y básica secundaria, esta medida se consigue del cuestionario de Acciones y Actitudes Ciudadanas de la parte no cognitiva de la prueba de Competencias Ciudadanas, 
conforme con el conversatorio, el ISCE tiene tanto de bueno como de malo; empero, una de las conclusiones importantes es que es preferible tener una medida, realizable de mejorar, que no poseerla.

Ahora lo bueno del índice es que se ha cimentado a partir de referentes internacionales, por ejemplo, se tomó como referente el índice desarrollado en Brasil. El índice compone un instrumento valioso por varios aspectos. En primer lugar, EI ISCE ha puesto a hablar a varios actores sobre el tema de la calidad educativa. Esto es fundamental en un instante donde la educación ha ganado terreno en varios escenarios. Segundo, EI ISCE es factible de comunicar en la medida que logra compendiar en un único número varias dimensiones de la calidad educativa. Tercero, los componentes del índice no solo calculan el valor absoluto de los resultados sino el progreso. Cuarto, el ISCE no solo mide resultados en pruebas sino además la aprobación y ambiente escolar, lo que indica una medida más completa de calidad. Quinto, es la primera vez que entra en las mediciones el desempeño de los estudiantes de 3, 5 y 9 , esto puede hacer que los rankings muestren nombres de colegios diferentes a los que estamos regularmente acostumbrados a ver. (Bayona, 2016), citado por (Díaz, 2018)

Entre tanto lo malo del índice es que tiene tanto limitaciones como inconvenientes. Primeramente, las medidas utilizadas aún son transitorias y el $80 \%$ del índice está enfocado en pruebas saber. Segundo, la prueba de competencias ciudadanas no tiene mucha variabilidad (todos contestan cerca de lo mismo), lo que muestra que esa parte del indicador no contribuye al índice. Tercero, el índice se orienta simplemente en las áreas de español y matemáticas a pesar de que otras áreas son evaluadas. Cuarto, usar un indicador recurrentemente para medir y tomar medidas de política pública puede traer consigo conductas no deseadas (ley de Campell). Por ejemplo, en países donde se ha encaminado en pruebas de español y matemáticas, se han evidenciado casos en los cuales se han limitado asignaturas de arte o de deportes para aumentar la intensidad de horas en las áreas evaluadas. Otra posible conducta no apetecida es que se le quiera hacer trampa al indicador. Un ejemplo simbólico se dio en Estados Unidos donde los maestros, quienes aplicaban el examen de sus estudiantes como sucede aquí, cambiaban las respuestas de sus estudiantes para que ellos lograran mejores notas. Esto lo hacían pues parte de su salario dependía de los resultados de los estudiantes. (Bayona, 2016), citado por (Díaz, 2018).

$Y$ lo feo en el corolario es que los investigadores presentaron algunos casos que, aunque no son una prueba irrebatible, nos generan preguntar sobre si podría actuar la ley de Campell al usar el ISCE. En primer lugar, se halló en algunas instituciones que el valor del índice para el año 2014 y reportado en 2015 difería de la cifra para el mismo año pero registrada en el 2016. En segundo lugar, se evidenció que existen instituciones con desempeño promedio en 2014 que obtuvieron que todos sus estudiantes evaluados pasaran al top de la tabla. Lo insólito de la situación es que el número de estudiantes que presentaron la prueba en algunas de esas escuelas bajó dramáticamente. (Bayona, 2016), citado por (Díaz, 2018).

Según (Robles, 2010), citado por (Díaz, 2018). Para el INEE la calidad de la educación es un elemento multidimensional macroscópico que no sólo tiene que ver con los niveles de aprendizaje de los estudiantes. Incluye al menos las siguientes ocho dimensiones: equidad: medidas y apoyos específicos acordes con las necesidades específicas de quienes los solicitan, para que los objetivos educativos sean logrados por el mayor número permisible de

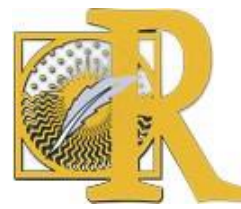


estudiantes; relevancia y pertinencia: cualidad de que el currículo distribuido refleje las necesidades de la sociedad y de los estudiantes; eficacia interna: coherencia entre los itinerarios escolares observadas y los objetivos encauzados a lograr que la más alta proporción de destinatarios permita a la escuela y permanezca en ella avanzando con los ritmos predichos hasta su egreso; eficacia externa: alcance de logros en el aprendizaje en consonancia con los objetivos previstos en el currículo; impacto: correspondencia ajustada entre los efectos de largo plazo de la educación y el objetivo de conseguir el aprovechamiento duradero de los aprendizajes, lo mismo que su traducción en conductas con valor social; suficiencia: correspondencia entre la cantidad y competitividad de los recursos humanos y materiales con los requerimientos pedagógicos y de equipamiento de las escuelas; eficiencia: utilización óptima de los recursos en el logro de los resultados conseguidos.

Según (Eurydice, 2012), citado por (Díaz, 2018) en Europa en 2010, los Estados Miembros y la Comisión Europea pactaron incluir el ámbito de la educación y la formación como un elemento clave en 'Europa 2020', la estrategia de la UE para un progresión inteligente, llevadero e inclusivo durante la próxima década. Esta estrategia se afirma, a su vez, en el Marco Estratégico para la Cooperación Europea en el espacio de la Educación y la Formación ("ET 2020") y sus cuatro objetivos transcendentales a largo plazo. El Marco Europeo se configura como la base para la colaboración europea en el ámbito de la educación y la formación, de forma que se muda en una contribución de primer orden para lograr los objetivos más amplios señalados en la estrategia "Europa 2020". Para llevar a cabo el alcance de los avances hacia la obtención de dichos objetivos es fundamental instalar de una serie de indicadores eficaces y armonizados.

\section{Población.}

Esta investigación se centró en los datos de excelencia en el trienio 2016 a 2018 para los estudiantes de grado noveno de la Institución Educativa General Santander del municipio de Soacha-Cundinamarca. En la tabla 1, se puede apreciar los resultados del ISCE para básicasecundaria.

\section{Cálculo del componente desempeño.}

El Puntaje promedio de la Prueba Saber $9^{\circ}$ en las áreas de Lenguaje y Matemáticas. La escala de valores va de 100 a 500, siendo 500 el puntaje promedio más alto permitido. El ejercicio lo realizaremos para el año 2018, para ello utilizaremos la información relacionada en la tabla 1. 
Tabla 1. Índice Sintético de Calidad Educativa (ISCE). Básica-Secundaria

\begin{tabular}{|c|c|c|c|c|c|}
\hline tre & \multicolumn{5}{|c|}{ Básica - Secundaria } \\
\hline Año & Desempeño & Progreso & Eficiencia & $\begin{array}{l}\text { Amblente } \\
\text { escolar }\end{array}$ & ISCE \\
\hline 2018 & 2.49 & 0.66 & 0.98 & 0.75 & 4.89 \\
\hline 2017 & 2.51 & 0.48 & 0.96 & 0.75 & 4.70 \\
\hline 2016 & 2.34 & 0.14 & 0.97 & 0.76 & 4.20 \\
\hline
\end{tabular}

Fuente: (MEN, 2018).

Para el cálculo del componente desempeño para el año 2017, se toman los valores para el año 2017 de la figura 1.

Figura 1. Puntaje promedio de las Pruebas Saber $9^{\circ}$ en las áreas de Lenguaje y Matemáticas. La escala de valores es de 100 a 500 .

\section{Grado Noveno}

\section{Lenguaje}

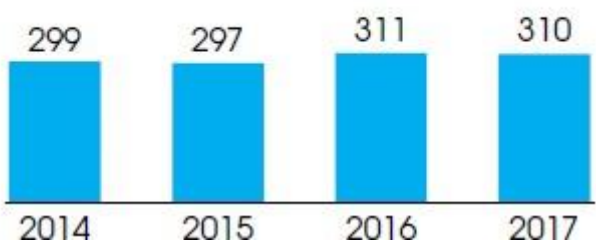

Matemáticas

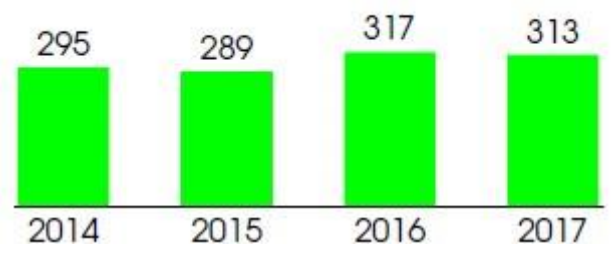

Fuente: (MEN, 2018)

Se suman los valores para lenguaje y matemáticas (2017), posteriormente se realiza la siguiente regla de tres:

Fecha de recepción: 11-06-2018 Fecha de aceptación: 31-05-2019 
Lenguaje $310+$ matemáticas $313=623$

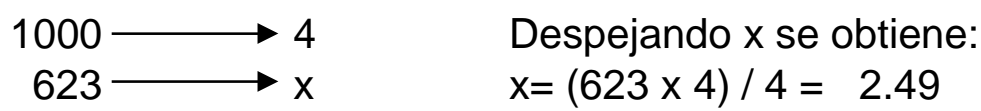

El dato 2.49 es que aparece en la Tabla 1, en el componente desempeño para el año 2018, para los otros años se calcula con el mismo procedimiento que para el año 2018.

\section{Cálculo del componente progreso.}

Este componente tiene un peso de 40 por ciento del ISCE; es decir que el máximo valor posible de obtener es 4 . Progreso tiene dos partes La figura 2, refleja que debemos sumar cuatro resultados en Primaria, dos en Secundaria y sólo uno en Media.

Figura 2. Estructura de los grados y áreas y su respectivo peso en cada nivel educativo

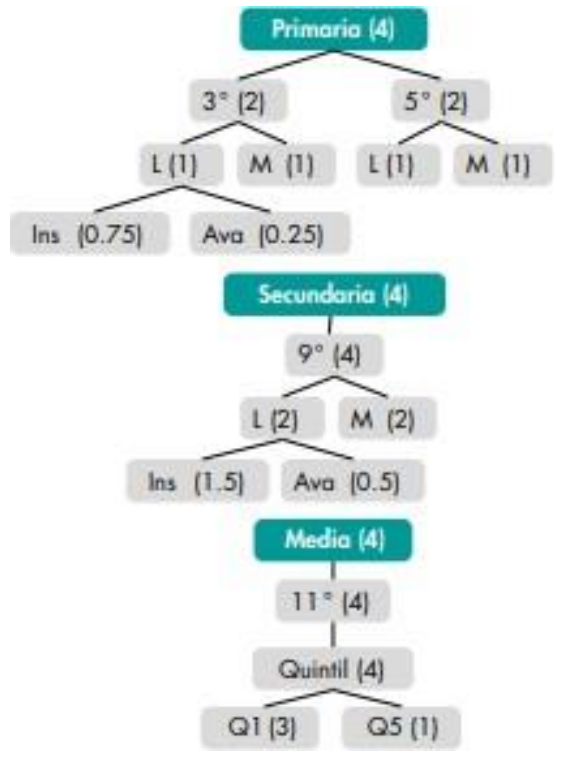

Primera parte: Nivel de desempeño Insuficiente La primera parte del componente Progreso, que pesa el 75 por ciento del componente es el cambio porcentual de un año a otro en el porcentaje de estudiantes en el nivel de desempeño Insuficiente. Para un grado y área, en el caso del ISCE 2018, hacemos el siguiente cálculo:

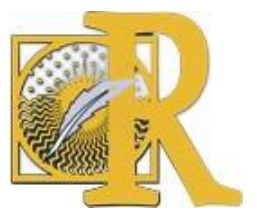

Fecha de recepción: 11-06-2018 Fecha de aceptación: 31-05-2019 


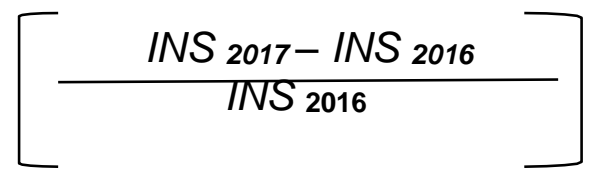

Reemplazando en la fórmula (1), obtenemos:

Lenguaje:

$$
\left[\frac{7-10}{10}\right]^{*(-1.5)=0.4}
$$

Matemáticas: $\quad\left[\frac{12-14}{14}\right]{ }^{*}(-1.5)=0.21$

Al sumar los dos componentes:

$$
\text { Insuficiente }=0.45+0.21=0.66
$$

Donde INS $i$ es el porcentaje de estudiantes en el nivel de desempeño Insuficiente de un grado y área en los años 2016 y 2017. Multiplicamos el cambio porcentual por 0.75 para darle el peso de 75 por ciento y lo multiplicamos por -1 para invertir el signo.

Para el nivel escolar Secundaria hay que calcular dos veces la Formula (1). En las áreas de lenguaje y matemáticas.

Segunda parte: Nivel de desempeño Avanzado. La segunda parte del componente Progreso, que pesa el 25 por ciento del componente, es el cambio porcentual de un año a otro en el porcentaje de estudiantes en el nivel de desempeño Avanzado. Para un grado y área, en el caso del ISCE 2018, hacemos el siguiente cálculo:

$$
\left.\left[\frac{\text { AVAN }_{2017}-\operatorname{AVAN}_{2016}}{100-\text { AVAN } 2016^{*}}\right] * .5\right)
$$

Reemplazando en la fórmula (2), obtenemos:

Lenguaje: $\left[\frac{2-6}{100-6}{ }^{*}(0.5)=0.0\right.$ $\left[\begin{array}{c}2-6 \\ 100-6\end{array}\right]$

Matemáticas: $\quad\left[\begin{array}{c}3-3 \\ 100-3\end{array}\right] *(0.5)=0.0$ 
Al sumar los dos componentes:

$$
\text { Avanzado9 }=0.0+0.0=0.0
$$

De los datos de la figura 3, se observa que, AVAN es el porcentaje de estudiantes en el nivel de desempeño Avanzado en un grado y área en los años 2017 y 2016. Multiplicamos el cambio porcentual por 0.5 para darle el peso de 25 por ciento a esta parte del componente. A diferencia de la primera parte del componente, aquí no multiplicamos por -1. Es decir, un aumento en el porcentaje de estudiantes de un año a otro en el nivel de desempeño Avanzado genera un aumento en el componente Progreso. A diferencia de la primera parte del componente, el denominador de la formula (2) es 100 menos el porcentaje en el año inicial. Esto se debe a que de esta forma el cambio porcentual mantiene la escala en la parte positiva de los valores alcanzables. Consecuentemente, la máxima calificación posible de obtener es 1.

Ahora tenemos todos los componentes necesarios para calcular

Progreso en básica secundaria: $0.66+0.0=0.66$

Figura 3. Niveles de desempeño de la Prueba Saber $9^{\circ}$ en las áreas de Lenguaje y Matemáticas. La escala de valores es de $0 \%$ a $100 \%$.

\section{Grado Noveno}

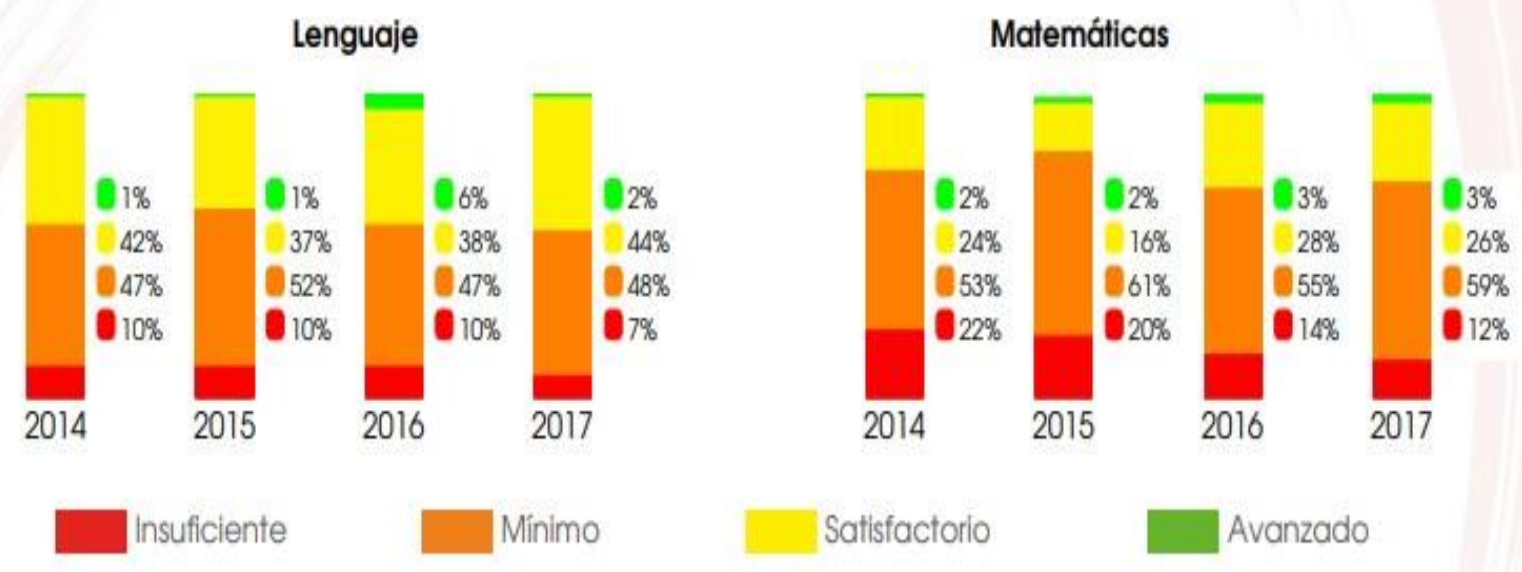

Fuente: (MEN, 2018).

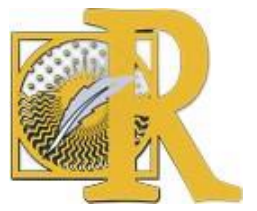




\section{Cálculo del ambiente escolar}

Ambiente Escolar El componente Ambiente Escolar pesa 10 por ciento del ISCE en los niveles educativos Primaria y Secundaria; es decir que el máximo puntaje posible de obtener es 1. El nivel educativo Media no tiene este componente.

En el ISCE 2015, Eficiencia provino del año 2013 y en el ISCE 2016 provino del año 2015. La información para el componente Ambiente Escolar proviene del cuestionario de Acciones y Actitudes Ciudadanas de la parte no cognitiva de la prueba de Competencias Ciudadanas que toman los estudiantes de los grados Quinto y Noveno cuando realizan la prueba Saber $3^{\circ}, 5^{\circ}$ y $9^{\circ}$.

El componente Ambiente Escolar lo construimos basados en dos medidas de esta prueba: Ambiente en el Aula y Seguimiento al Aprendizaje. Esta información está en un escala de 100 a 200 y es particular para Lenguaje y Matemáticas; por lo tanto para los grados Quinto $Y$ Noveno, tenemos cuatro escalas diferentes. Promediamos estas cuatro mediciones y dividimos por 200, para obtener una escala de 0 a 1.

En los reportes de Colombia Aprende publicados por el Ministerio de Educación Nacional aparece una calificación para cada una de las dos partes que componente el Ambiente Escolar. Para recuperar el resultado final de Ambiente Escolar debemos seguir el siguiente procedimiento:

$$
\frac{A m b+\operatorname{Seg}+200}{400}
$$

Para este caso sería:

$$
\frac{50+50+200}{400}=0.75
$$

En donde Amb es Ambiente en el aula y Seg es Seguimiento al Aprendizaje. Amb y Seg están sin el 100 que divide el resultado en el reporte de Colombia Aprende.

\section{Cálculo de la Eficiencia.}

Según el (ICFES, 2016). El componente Eficiencia pesa 10 por ciento del ISCE en el nivel educativo Secundaria; quiere decir que el máximo puntaje posible de obtener es 1.

"Eficiencia es la tasa de aprobación al siguiente año escolar de cada nivel educativo. La tasa de aprobación, junto con otros indicadores de educación, está en el Sistema Nacional de Indicadores de Educación Preescolar, Básica y Media. Este componente lo entrega el Ministerio de educación Nacional al Icfes. La tasa de aprobación ya está en una escala de 0 a 1". (ICFES, 2016)

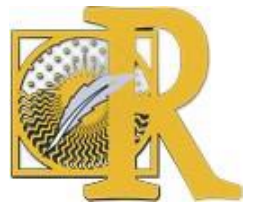


Figura 4. Resumen del Índice Sintético de Calidad Educativa (ISCE) del cuatrienio 2015-2018 y sus respectivos componentes.

\section{ISCE BASICA-SECUNDARIA 2016-2018}

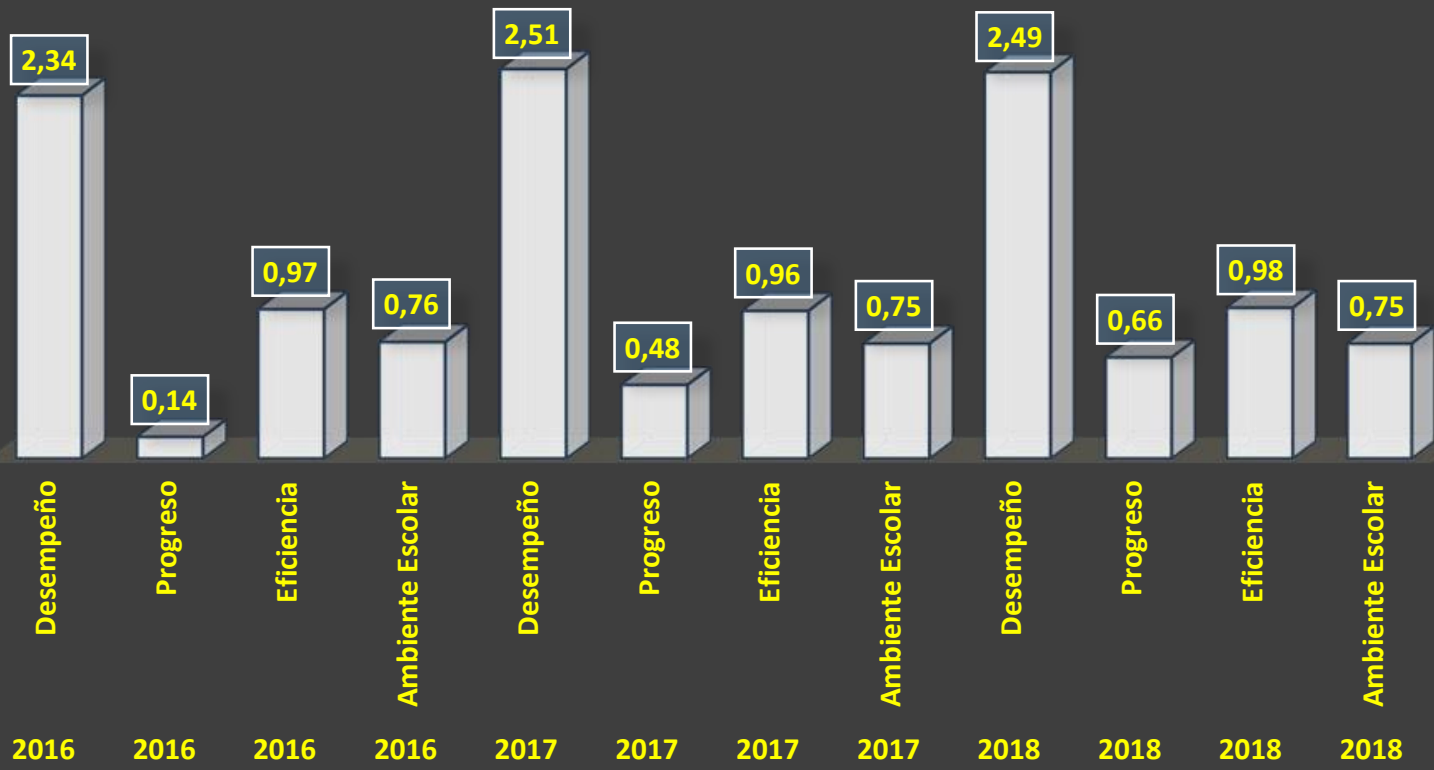

Fuente: Elaboración propia del autor.

En la figura 4, se observan los diferentes componentes del Índice Sintético de Calidad Educativa (ISCE), para los años 2016 al 2018. Para el componente desempeño se aprecia que para el 2015 fue de 2,34, presentando un leve aumento en el 2016 al pasar 2,51, esto debido por la mejora en la calificación en Lenguaje y matemáticas. En el componente progreso de aprecia un incremento desde año 2016 con un 0,14, en el 2017 de ,48 y en el 2018 del 0,66. Esto debido a la disminución de estudiantes en el nivel insuficiente. Para el componente eficiencia el dato permanece constante 0,97 en el 2016, 0,96 en el 207 y 0,98 en el 2018. De igual forma se observa para el componente ambiente escolar con 0,76 en el 2016, 0,75 en el 2017 y 0,75 en el 2018.

Para el caso del Índice Sintético de Calidad Educativa (ISCE), se observa un incremento progresivo, en el año 2016 fue de 4,20, en el 2017 de 4,70 y en el 2018 de 4,89. 


\section{Conclusiones.}

En países vecinos como Brasil, se utiliza el Índice de Desenvolvimiento de Educación Básica (IDEB) se fundamente en base en el producto de dos componentes: flujo escolar y rendimiento. El primero tiene que ver con el número de estudiantes que aprueban el curso; el segundo, está conexo con los resultados de los estudiantes en la Prueba Brasil. (Remolina, 2015).

Según (Francia, 2017).En el 2015 se obtuvieron los siguientes resultados según el IDEB, en la escuela secundaria de 3,7, en promedio de 4,6. Ahora comparado este resultado de Brasil con los resultados ISCE para la Institución Educativa General Santander para básica Secundaria fue de 4,89 en el 2018, se aprecia ligeramente por encima con el IDEB en Brasil. En lo concerniente para Institución Educativa General Santander, se aprecia un incremento en su ISCE a partir del año 2016, esto debido a los planes de mejoramiento que la institución ha implementado año tras año.

Comparemos el dato del ISCE con una institución educativa en Pasto-Nariño, que consigue el nivel mínimo con un promedio de 4,12, apartado del promedio nacional que es de 4,93 y más aún del promedio de la entidad certificada, municipio de pasto, que se ubica en 6,30 , asimismo plantea una eficiencia que se expresa en el nivel de promoción de estudiantes de grado agrado, dentro de la básica secundaria $83 \%$, lo cual enuncia que en la promoción es alta frente a los niveles de calidad que se expresa en el desempeño de los estudiantes dentro de la pruebas saber, que es minúscula, para este nivel. (Franco, 2016)

Para tratar de mejorar el ISCE, se pueden mejorar las prácticas de aula en matemáticas, ejemplo de ello tenemos los trabajos realizados por (Díaz, 2016a; 2016b; 2017a; 2017b), con el uso de simuladores, logrando evidenciar una mejora en la enseñanza de las matemáticas, además se debe tener una formación permanente de los docentes en el conocimiento y apropiación de las tecnologías de la información y la comunicación. (Díaz, 2017c; 2017d).

Según (Universidad del Norte, 2015). Los ambientes de aprendizaje habrán de promover búsquedas críticas, donde la formación magistral sea reemplazada por opciones que conduzcan a una reflexión sobre la práctica, edificada desde la experiencia de los participantes; donde el lugar de alguien que da soluciones sea depuesto por una cimentación colectiva que reconoce los efectos diferentes que tienen las estrategias pedagógicas en cada grupo social.

Otra alternativa para mejorar el desempeño, el progreso, la eficiencia y el ambiente de aula son las salidas pedagógicas, de acuerdo con (Díaz, 2016c; 2016d), son una estrategia de enseñanza que crea en los estudiantes una visión general de los conceptos adquiridos en clase, además de propiciar el aprendizaje dinámico, ofrecer nuevos espacios para la enseñanza, la comprobación, el análisis y la construcción del conocimiento y permitir el contacto de los estudiantes con aptitudes científicas e investigativas.

También es preponderante, justificar planeaciones docentes pertinentes al contexto de la institución educativa acordes con los criterios propuestos por el MEN. Conformar de equipos interdisciplinarios que sean líderes de los programas de casos especiales de medio y bajo rendimiento. Realizar formaciones relacionados con el consolidamiento del valor de la autoestima y comunicación asertiva que accedan en el estudiante desenvolver más su inteligencia emocional y afectiva.

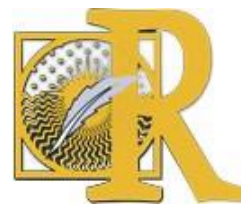




\section{Referencias Bibliográficas.}

Bayona, H. (2016). Lo bueno, lo malo y lo feo del Índice Sintético de la Calidad Educativa (ISCE). Recuperado de: http://lasillavacia.com/silla-llena/red-de-la-educaci-n/historia/lobueno-lo-malo-y-lo-feo-del-ndice-sint-tico-de-la-calidad

Díaz, J. (2016a). Soporte técnico de simulación Phet en la enseñanza y aprendizaje de fracciones equivalentes. Revista de investigaciones Universidad del Quindío, 28 (2), 31-41. Recuperado

de: http://blade1.uniquindio.edu.co/uniquindio/revistainvestigaciones/adjuntos/pdf/f30d Galeras1-23-33.pdf

Díaz, J. (2016b). Aplicación PhET: estrategia de enseñanza-aprendizaje de fracciones equivalentes. Revista Criterios, 23 (1), 111-123. Recuperado de. http://www.umariana.edu.co/ojs-ditorial/index.php/criterios/article/view/1193

Díaz, J. (2016c). Planetario: Estrategia de aprendizaje. Revista Fedumar Pedagogía y Educación, 3(1), 117-125. (2016). Recuperado de: http://www.umariana.edu.co/ojseditorial/index.php/fedumar

Díaz, J. (2016d). Clubes de Astronomía: Didáctica de enseñanza de la Ciencia y la Investigación. Revista Fedumar Pedagogía y Educación, 3(1), 127-137 (2016). En línea http://www.umariana.edu.co/ojs-editorial/index.php/fedumar.

Díaz, J. (2017a). Importancia de la simulación Phet en la enseñanza y aprendizaje de fracciones equivalentes. Revista Educación y Desarrollo Social. Vol.11, No1. (2017). En línea: https://revistas.unimilitar.edu.co/index.php/reds/article/view/2011

Díaz, J. (2017b) Simulador Applet Descartes: Como didáctica de enseñanza de la función cuadrática. Revista Innova Research Journal, 2 (8). Recuperado de: http://www.journaluidegye.com/magazine/index.php/innova/article/view/256

Díaz, J. (2017c). Conocimiento y apropiación de las tecnologías de la información y la comunicación (TIC) en la formación docente. Revista Actualidades Pedagógicas. Número $69,121-131$.

Recuperado

de:

https://revistas.lasalle.edu.co/index.php/ap/article/view/4066/3198

Díaz, J. (2017d). Formación de Docentes en el Uso y Apropiación de las Tic. Revista Innova Research Journal. Vol.2 Número 9. (2017). Recuperado el 15 de octubre de 2017 de: http://www.journaluidegye.com/magazine/index.php/innova/article/view/257/398

Díaz, J (2018). Análisis de los resultados del Índice Sintético de la Calidad Educativa (ISCE) 2017 de las Secretarías de Educación Certificadas de Colombia. Revista: International Journal of Educational Research and Innovation (IJERI), 10, 331-341

El Tiempo. (2017). Colombia superó las metas en educación para 2017. Recuperado el 20 de mayo de 2017 de: http://www.eltiempo.com/vida/educacion/resultados-del-indicesintetico-de-calidad-educativa-2017-86552

Eurydice. (2012). Cifras clave de la educación en Europa 2012. Recuperado de: http://eacea.ec.europa.eu/education/eurydice/documents/key data series/134ES.pdf 
Francia, L. (2017). Ideb: Aprende todo sobre el Índice de Desarrollo de la Educación Básica. Recuperado de: http://appprova.com.br/ideb/

Franco, R. (2016). Impactos de la política de calidad Educativa, en los niveles de educación básica secundaria y media de la Institución Educativa el Encano, Municipio de Pasto. Recuperado http://stadium.unad.edu.co/preview/UNAD.php?url=/bitstream/10596/8601/1/36757491.pdf Godoy, S. (2015). Índice Sintético de Calidad Educativa- ISCE. Recuperado el 20 de mayo de 2017 de http://www.ucc.edu.co/cali/prensa/2016/Publishinglmages/Paginas/Jornada-deCalidad-Educativa-en-la-U Instituto Colombiano para el fomento de la Educación Superior. (2016). ISCE: Guía Metodológica. Recuperado de: http://aprende.colombiaaprende.edu.co/ckfinder/userfiles/files/Gu\%C3\%ADa\%20Metodol\% C3\%B3gica ISCE.pdf Jaramillo, J. (2017). Siempre día-e. Recuperado de: http://aprende.colombiaaprende.edu.co/siemprediae Ministerio de Educación Nacional. (2017). ¿Qué es el índice sintético de calidad educativa (ISCE)? Recuperado de: http://www.colombiaaprende.edu.co/html/micrositios/1752/articles349835 quees.pdf

Remolina, J. (2015). La calidad sintética de los indicadores educativos. Recuperado de: https://www.las2orillas.co/la-calidad-sintetica-de-los-indicadores-educativos/

Robles, H. (2010). El Sistema de Indicadores Educativos del Instituto Nacional para la Evaluación de la Educación. Recuperado el 14 de octubre de 2017 de: http://publicaciones.inee.edu.mx/buscadorPub/P1/C/224/P1C224 06E06.pdf

Universidad del Norte. (2015). Propuesta de Evaluación con Carácter Diagnóstico Formativa ECDF.

Recuperado

de:

http://aprende.colombiaaprende.edu.co/sites/all/modules/mapa/docs/curso ECDF/UNINOR TE curso\%20ECDF.pdf

Zambrano, M (2015). Alcances e inconsistencias del Índice Sintético de Calidad Educativa (ISCE), diseñado y aplicado por el Ministerio de Educación de Colombia en el año 2015. Recuperado de: http://acofade.org/documentos/notiacofade/Indice\%20sinte\%C3\%ACtico\%20de\%20calidad \%20educativa MEN Colombia.pdf 